
\title{
25 Research Soure \\ Clinical T category for lung cancer staging: A pragmatic approach for real-world practice
}

\section{Yeonu Choi}

Samsung Medical Center

\section{Sun-Hyung Kim}

Samsung Medical Center

Ho Yun Lee ( $\nabla$ hoyunlee96@gmail.com )

Sung Kyun Kwan University School of Medicine at Samsung Medical Center Cancer Center https://orcid.org/0000-0001-9960-5648

\section{Sang-Won Um}

Samsung Medical Center

Ki Hwan Kim

Sungkyunkwan University

Yeonseok Choi

Samsung Medical Center

\section{Sung Goo Park}

Kangbuk Samsung Medical Center

Insuk Sohn

Samsung Medical Center

Hye Seung Kim

Samsung Medical Center

\section{Research article}

Keywords: lung adenocarcinoma (ADC), neoplasm staging, computed x-ray tomography, observer variation

Posted Date: February 7th, 2020

DOI: https://doi.org/10.21203/rs.2.22833/v1

License: (c) (i) This work is licensed under a Creative Commons Attribution 4.0 International License. Read Full License 
Version of Record: A version of this preprint was published at Thoracic Cancer on October 19th, 2020. See the published version at https://doi.org/10.1111/1759-7714.13701. 


\section{Abstract}

Background : To determine which components should be measured and which window settings are appropriate for computerized tomography (CT) size measurements of lung adenocarcinoma and to explore interobserver agreement and accuracy according to the 8th edition of TNM staging. Methods : One hundred sixty-five surgically resected lung adenocarcinomas earlier than stage 3A were included in this study. One radiologist and two pulmonologists independently measured the total and solid sizes of components of tumors on different window settings and assessed solidity. CT measurements were compared with pathologic size measurements. Results : In categorizing solidity, $25 \%$ of the cases showed discordant results among observers. Measuring the total size of a lung adenocarcinoma predicted pathologic invasive components to a degree similar to measuring the solid component. Lung windows were more accurate (intraclass correlation $[I C C]=0.65-0.81$ ) than mediastinal windows (ICC $=0.20-$ 0.72) at predicting pathologic invasive components, especially in a part-solid nodule. Interobserver agreements for measurement of solid components were good with little significant difference (lung windows, ICC $=0.89$; mediastinal windows, ICC $=0.91$ ). A high level of interobserver agreement was seen between the radiologist and the pulmonologists and between residents versus a fellow on different windows. Conclusions : A considerable percentage (25\%) of discrepancies was encountered in categorizing the solidity of lesions, which may decrease the accuracy of measurements. Lung window settings may be superior to mediastinal windows for measuring lung adenocarcinomas, with comparable interobserver agreement and moderate accuracy for predicting pathologic invasive components. Keywords : lung adenocarcinoma (ADC), neoplasm staging, computed x-ray tomography, observer variation

\section{Background}

The 8th edition of the TNM staging of lung cancers was recently proposed by the International Association for the Study of Lung Cancer (IASLC). For clinical T staging of subsolid nodules, the IASLC recommended measuring the long axis of the largest solid portion on lung windows to reflect the invasive component as close as possible to the pathologic T descriptor [1-7].

However, in the real-world practice, even radiologists, when classifying lesions by a clinical T descriptor of the 8th version of lung cancer staging, experience substantial numbers of atypical cases that are not clearly shown as a lesion of the central solid component with peripheral ground-glass opacity (GGO), often leading to difficulty on categorization of such ambiguous cases. Thus, various measurement approach dealing with diverse radiologic situations regarding subsolid nodule need to be compared comprehensively.

The purpose of this study was to 1) determine the degree of difference among categorizations of solidity by observers, 2) determine which components to measure, 3) identify which window settings to use, and 4) determine if there is difference between measurements by radiologists and pulmonologists. Ultimately, we hoped to identify the most appropriate way to measure various subsolid lesions. 


\section{Methods}

This retrospective study was approved by the institutional review board of our institution (IRB no. 201709-045); informed consent for using clinical and imaging data of the patients was waived.

\section{Study population}

We identified all cases with surgically resected ADC in the lung cancer registry of Samsung Medical Center from 2009 through 2016 and that satisfied the following inclusion criteria: confirmed ADC; earlystage, i.e., stage I, II, or IIIA according to the 8th version staging [1]; and presence of a $2 \mathrm{~mm}$ or less CT image performed before surgery. Exclusion criteria were history of previous radiation or chemotherapy.

\section{Image acquisition}

CT images were obtained with the following parameters: (field of view, 30 to $36 \mathrm{~cm}$; beam pitch, 1.35 or 1.375; gantry speed, 0.5 or 0.6 second per rotation; $120 \mathrm{kVp} ; 150-200 \mathrm{~mA}$; and reconstruction interval, $1-$ $2 \mathrm{~mm}$ ). Details are described in Supplemental Data 1.

\section{CT interpretation}

Three independent observers retrospectively evaluated the CT scans of the ADC cases. One observer was a radiology resident (observer 1 ). The others were physicians in pulmonology(observer 2, a resident; observer 3 , fellow).

The observers evaluated the solidity of the tumor in a lung window by visual inspection and categorized it as pure ground-glass nodule (GGN), part-solid, or solid nodule.

The sizes of the total and solid components of the ADC with different windows were evaluated by each observer as follows: 1) total area of the lesion in the lung window (total-lung area), 2) total area of the lesion in the mediastinal window (total-media area), 3) maximal diameter of the total lesion in the lung window (total-lung $M$ ), 4) maximal diameter of the total lesion in the mediastinal window (total-media M), 5) maximal diameter of the solid component in the lung window (solid-lung $M$ ), and 6) maximal diameter of the solid component in the mediastinal window (solid-media M).

Measurements were performed on 3-dimensional reconstruction images. And the sections and planes that displayed the largest tumor diameter were selected. If a part-solid nodule had several internal solid components, we measured the maximal diameter of the solid component by adding each measurable solid component.

\section{Comparison of CT-pathological size measurement}

We compared CT and pathologic sizes in two different ways. First, the total area of the tumor on CT was compared with the total area of the lesion in pathology (Fig. 1A). Second, the maximum diameter of the tumor was compared with the invasive pathologic component, as the pathologic report contained only the single maximum diameter of the invasive component (Fig. 1B). An absolute difference value was 
obtained by subtracting the maximum CT diameter of the solid component from the pathologic invasive size.

\section{Statistical analysis}

A statistical analysis was executed using SAS version 9.4 (SAS Institute, Cary, NC) and R 3.5.1 (Vienna, Austria; http://www.R-project.org).

To compare radiologic and pathological size measurements of ADCs, Bland-Altman plots with 95\% confidence intervals (Cls) and intraclass correlation coefficient (ICC) values were assessed. To determine the agreement of solidity categorization of each observer, weighted Cohen's kappa values were assessed. A Wilcoxon signed rank test was performed to compare the difference between the solid component size and pathologic invasive component size according to window settings.

Interobserver and intraobserver agreement for size measurements of the ADCs was assessed using ICC values. Significance was set at $p<0.05$.

\section{Results}

\section{Characteristics of the patients}

A total of 165 patients with 165 ADCs satisfied our inclusion criteria (79 men and 86 women, mean age 61 years; range 36-82 years). All patients underwent surgery. The mean time between CT and surgery was 15 days ( $S D=18$ days; range $=0-118$ days). Additional details of patient characteristics are summarized in Supplemental table 1.

\section{Agreement of solidity categorization}

Each observer categorized the lung lesion as pure-GGN, part-solid, or solid nodule. Overall, $75 \%(n=124)$ of the lesions exhibited concordant results among the three observers, and 25\% ( $n=41)$ showed discrepancies, with at least one classified differently (Table 1). The ratio of discordant cases ranged from $13-22 \%$, based on a comparison between two observers. 
Table 1

Summary of solidity categorization among three observers.

\begin{tabular}{|c|c|c|c|c|c|c|}
\hline \multirow[t]{2}{*}{$\begin{array}{l}\text { Solidity } \\
\text { classification }\end{array}$} & $\begin{array}{l}\text { Observer } \\
1\end{array}$ & $\begin{array}{l}\text { Observer } \\
2\end{array}$ & $\begin{array}{l}\text { Observer } \\
3\end{array}$ & $\begin{array}{l}\text { Concordant } \\
\text { cases } \\
(n=124,75 \%)\end{array}$ & \multicolumn{2}{|c|}{$\begin{array}{l}\text { Discordant cases } \\
(n=41,25 \%)\end{array}$} \\
\hline & \multicolumn{3}{|c|}{ No. of lesions $(n=165)$} & \multicolumn{3}{|c|}{ No. of lesions $(n=165)(\%)$} \\
\hline Pure-GGN & 8 & 17 & 24 & 8 (5) & $7(4)^{*}$ & \\
\hline Part-solid & 109 & 82 & 95 & $73(44)$ & $\begin{array}{l}11 \\
(7)^{\dagger}\end{array}$ & $\begin{array}{l}18 \\
(11)^{\ddagger}\end{array}$ \\
\hline Solid & 48 & 66 & 46 & $43(26)$ & & $5(3)^{a}$ \\
\hline
\end{tabular}

Agreement of solidity categorization by weighted Cohen's kappa value was generally good $(\mathrm{k}=0.65-0.78$, $95 \% \mathrm{Cl}=0.55-0.87)$. Weighted Cohen's kappa values and $95 \% \mathrm{Cl}$ between two observers were as follows: observer 1 and $2(\mathrm{k}=0.65,95 \% \mathrm{Cl}=0.55-0.76)$, observer 1 and $3(\mathrm{k}=0.78,95 \% \mathrm{Cl}=0.70-0.87)$, observer 2 and $3(k=0.67,95 \% \mathrm{Cl}=0.57-0.77)$

To determine if any specific patterns caused the differences in solidity categorization, we further analyzed 41 discordant cases. Of 41 cases, 18 cases (44\%) were difficult to distinguish between pureGGN and part-solid nodule. And of 41 cases, 23 cases (56\%) were difficult to distinguish between partsolid and solid nodule.

Cases where pure-GGN and part-solid nodules needed to be distinguished divided into three categories. The first was subsolid nodules with CT features of borderline attenuation $(n=15,83 \%)$, which means a higher than usual density GGO in lung windows but not visible as a solid portion in mediastinal windows (Fig. 2A). In these cases, observers had difficulty classifying lesions as a pure-GGN or part-solid nodule. The second was subsolid lesions with entirely heterogeneous attenuation $(n=2,11 \%)$ that could not be classified into pure-GGN or homogeneous solid nodules (Fig. 2B). The last category was subsolid lesions with a smoothly transitional margin from GGO to dense solid attenuation $(n=1,6 \%)$, which means the lesion has no clear-cut margin between the dense solid component to background GGO (Fig. 2C).

In cases where part-solid and solid nodules needed to be distinguished, most of the cases difficult to distinguish were solid nodules with an adjacent small area of GGO $(n=18,78 \%)$ (Fig. 2D). Owing to mass effect or obstruction of bronchus, the peripheral GGOs could have confused observers.

\section{Accuracy of CT size measurement compared with pathology}

For accuracy compared with pathologic size, total lung area generally showed high ICC (0.89-0.91) between total pathologic areas of the lesion (Table 2). Total media area also showed moderate to high 
degree of accuracy compared with pathologic total area $(I C C=0.77-0.83)$. Solid-lung M showed moderate to high accuracy (ICC $=0.79-0.85)$ compared with the diameter of the pathologic invasive component. However, solid-media M showed poor to moderate accuracy $(I C C=0.58-0.75)$ between pathologic invasive components.

Table 2

Overall accuracy of CT measurements compared with pathologic size measurement of lung adenocarcinoma

\begin{tabular}{|lll|}
\hline Size measurement & ICC & $95 \% \mathrm{Cl}$ \\
\hline Total-lung area & $0.89-0.91$ & $0.86,0.94$ \\
\hline Total-media area & $0.77-0.83$ & $0.69,0.87$ \\
\hline Solid-lung M* & $0.79-0.85$ & $0.73,0.89$ \\
\hline Solid-media M* & $0.58-0.75$ & $0.48,0.81$ \\
\hline
\end{tabular}

The median of the absolute difference value (pathologic invasive size - maximal CT diameter of solid component) was $8 \mathrm{~mm}$ (interquartile range [IQR], 4 to 11) in the mediastinal window and $3 \mathrm{~mm}$ (IQR, - 7 to 17) in the lung window. The difference value of the solid component was significantly higher in mediastinal windows than lung windows in all observers $(p<0.0001)$.

To determine the effect of solidity on CT to predict pathologic tumor status, further analysis was carried out by layering according to solidity classification (Table 3). Measurement of solid nodules generally showed moderate to high accuracy for predicting both pathologic total area and invasive pathologic component $(I C C=0.73-0.94)$. Measurement of pure-GGNs showed poor accuracy for predicting pathologic total area (ICC $=0.0-0.52)$. 
Table 3

Accuracy of CT measurements compared with pathologic size measurement of lung adenocarcinoma: layering by solidity

\begin{tabular}{|c|c|c|c|c|c|c|}
\hline \multirow[t]{2}{*}{ Size measurement } & \multicolumn{2}{|l|}{ Pure-GGN } & \multicolumn{2}{|l|}{ Part-solid } & \multicolumn{2}{|l|}{ Solid } \\
\hline & ICC & $95 \% \mathrm{Cl}$ & ICC & $95 \% \mathrm{Cl}$ & ICC & $95 \% \mathrm{Cl}$ \\
\hline Total-lung area & $0.29-0.52$ & $0.02,0.90$ & $0.80-0.86$ & $0.71,0.91$ & $0.93-0.94$ & $0.88,0.97$ \\
\hline Total-media area & & & $0.35-0.55$ & $0.19,0.68$ & $0.89-0.94$ & $0.83,0.97$ \\
\hline Total-lung M* & $0.0-0.18$ & $0.0,1.0$ & $0.65-0.81$ & $0.53,0.87$ & $0.87-0.90$ & $0.79,0.94$ \\
\hline Total-media M* & & & $0.52-0.72$ & $0.36,0.81$ & $0.83-0.93$ & $0.74,0.96$ \\
\hline Solid-lung M* & & & $0.65-0.79$ & $0.52,0.85$ & $0.82-0.93$ & $0.72,0.96$ \\
\hline Solid-media $M^{\star}$ & & & $0.20-0.63$ & $0.07,0.73$ & $0.73-0.92$ & $0.60,0.95$ \\
\hline
\end{tabular}

For measurement of total area of part-solid nodules, the lung window demonstrated high accuracy (ICC = 0.80-0.86) for predicting pathologic total area. Otherwise, mediastinal window demonstrated poor accuracy (ICC $=0.35-0.55)$. In measurement of solid components in part-solid nodules, the lung window provided a superior, although moderate, degree of accuracy $(I C C=0.65-0.79)$ for predicting invasive pathologic components to the mediastinal window $(I C C=0.20-0.63)$.

We found no significant difference of accuracy for predicting pathologic invasive component between total-lung M (ICC = 0.65-0.81) and solid-lung M (ICC = 0.65-0.79).

Bland-Altman plots with $95 \%$ Cls of the difference between the size of the pathologic invasive component and the maximum diameter of solid components on CT are shown in Supplemental Figs. 1 and 2 .

\section{Interobserver and intraobserver agreement of radiologic size measurement}

ICC values of radiologic size measurement among three observers generally ranged from 0.89 to 0.97 , representing a high agreement (Table 4). No significant difference in interobserver agreement was seen for solid-lung M (ICC = 0.89) and solid-media M $(I C C=0.91)$. 
Table 4

Interobserver agreement of CT size measurement

\begin{tabular}{|lllllll|}
\hline $\begin{array}{l}\text { Size } \\
\text { measurement }\end{array}$ & \multicolumn{2}{l}{\begin{tabular}{l}
\multicolumn{2}{l}{ Total interobserver } \\
agreement
\end{tabular}} & \multicolumn{2}{l}{$\begin{array}{l}\text { Radiology vs. } \\
\text { pulmonology }\end{array}$} & \multicolumn{2}{l|}{$\begin{array}{l}\text { Resident vs. } \\
\text { fellow }\end{array}$} \\
\cline { 2 - 8 } & ICC & $95 \% \mathbf{C l}$ & ICC & $95 \%$ Cl & ICC & $95 \%$ Cl \\
\hline Total-lung area & 0.95 & $0.93,0.96$ & 0.96 & $0.95,0.97$ & 0.93 & $0.91,0.95$ \\
\hline Total-media area & 0.97 & $0.96,0.97$ & 0.97 & $0.97,0.98$ & 0.96 & $0.94,0.97$ \\
\hline Solid-lung M* & 0.89 & $0.87,0.92$ & 0.91 & $0.88,0.93$ & 0.90 & $0.90,0.94$ \\
\hline Solid-media $M^{*}$ & 0.91 & $0.89,0.93$ & 0.93 & $0.91,0.94$ & 0.89 & $0.86,0.92$ \\
\hline
\end{tabular}

We subdivided the group as department of radiology (observer 1) versus pulmonology (observers 2 and 3) and resident (observers 1 and 2) versus fellow (observer 3). Each subgroup analysis also showed high agreement in two groups: department analysis $($ ICC $=0.91-0.97)$ and education level analysis $($ ICC $=$ 0.89-0.96).

Although there was generally a high degree of agreement for CT measurement among observers, we conducted analyses of specific cases with a low degree of agreement. For measurement of solid-media $\mathrm{M}$, we found 7 outlier cases that showed significantly different values between observers. The most common one was lesions that had internal air density or an air-bronchogram (Fig. 3). On the mediastinal window, multiple scattered linear or nodular solid components made it difficult to measure consistently. In part-solid nodules with several internal solid components, interobserver agreement of solid component was lower (Fig. 4). Some cases also showed irregular, spiculated margins, which were difficult to measure point to point (Fig. 3). Adjacent vascular structures or pleural tagging caused confusion in some observers. One case showed a central location adjacent to hilar structures.

Intraobserver agreement for total area of the lesion in different windows ranged from 0.83 to 0.87 (95\% $\mathrm{Cl}=0.78-0.90)$. Intraobserver agreement for solid component of the lesion in different windows ranged from 0.87 to $0.97(95 \% \mathrm{Cl}=0.82-0.98)$, representing a high agreement.

\section{Discussion}

Interest in measuring the exact size of the solid component of lung adenocarcinoma on CT has increased because of the close relationship to prognosis [2-4, 8-10], and several studies have established standardized methods to evaluate solid components with different windows. The present study focused on the differences in solidity categorization between observers and which components and windows should be used to determine clinical T staging of ADC. 
Surprisingly enough, when categorizing the solidity of lesions, approximately $25 \%$ were atypical cases that caused discordant results among observers. Given that interobserver agreement has been reportedly low when classifying lesion types (pure-GGN, part-solid, or solid) in lung window-based assessment in several studies $[7,11]$, our results showed a generally good, but not exceptionally high, agreement among observers.

According to our analysis, three different radiologic situations made categorization difficult. First, subjective interpretation of borderline attenuating lesions in the lung window was the main cause of the discordancy (Fig. 2A). Because borderline attenuated lesions were more dense than usual GGOs, they caused confusion as to whether they should be classified as pure-GGN or part-solid nodules. In the Fleischner glossary, the solid component of a part-solid nodule should fulfill the criteria of consolidation. Fleischner recommendations advised mediastinal windows for evaluation of solid components, which usually exceed $-160 \mathrm{HU}[12,13]$. However, a study by Lee et al. suggested that a lower density range of 261 to $-160 \mathrm{HU}$ is more appropriate to describe an invasive tumor component [4]. There is therefore a question of whether these borderline attenuated lesions should be considered solid, and further discussion is warranted. Although they constitute relatively small portions, other lesions such as entirely heterogeneous lesions or lesions with smoothly transitional margins also caused inconsistencies among observers (Figs. 2B and 2C). All three types described above caused inconsistencies among observers, creating a considerable number $(25 \%)$ of discordant atypical cases. As inaccurate solidity categorization may affect the accuracy of measurements, visual assessment alone limits accuracy and consistent categorization.

Many groups have reported the possibility of using histograms and texture analysis to distinguish invasive ADC from pre-invasive ADC or MIA and to predict tumor metabolism or stages [14-17]. Ikeda et al. reported that the 75th percentile CT number of GGO lesions was the optimal CT number for differentiating atypical adenomatous hyperplasia and other histology using a CT number histogram [15]. Similarly, in categorizing borderline attenuated lesions or lesions with smoothly transitional margins, a histogram analysis may supply a more objective method for differentiating between pure-GGN and partsolid nodules. Also, texture analysis that includes entropy and uniformity could help categorize entirely heterogeneous attenuated lesions.

As to which components should be measured to predict invasive components and patient prognoses in part-solid nodules, recent studies have shown that solid components are more useful than total components [2, 8-10]. However, our study demonstrated that the total size measurement is no worse than solid component measurements at predicting invasive components. This is likely because we included a variety of lesions that contained relatively large solid components, while most previous studies targeted small tumors $(\leq 3 \mathrm{~cm})$ that had a substantial ground-glass or lepidic component. Further study is needed to determine which component should be measured in the case of larger tumors or tumors composed predominantly of invasive components with a minor ground-glass component. 
In terms of the reproducibility of the measurement, we compared interobserver agreements among radiologists and pulmonologists with respect to the total and solid component measurements between lung and mediastinal windows. We found no significant differences in interobserver agreement for both solid and total size measurements at different windows. We also found a very good degree of interobserver agreement between the radiologist and pulmonologists and between residents versus fellow. Lee et al. reported that both windows could be applied to solid component measurement without a significant difference in the case of MIA [5]. Yoo et al. reported no significant difference in interobserver agreement among five readers for solid component measurements between two windows in the case of subsolid nodules with solid components smaller than $8 \mathrm{~mm}$ [18]. Our results are consistent with these previous studies. However, unlike previous studies that targeted only patients with solid components smaller than $8 \mathrm{~mm}$, our study targeted various sizes of ADC and included more patients, similar to a real clinical setting. Our results reconfirmed and strengthened the previous findings by larger number and a wide range of lesions. We also confirmed that a pulmonologist can measure as well as a radiologist.

In terms of accuracy of size measurement, the lung window demonstrated greater accuracy compared with mediastinal window, especially in the case of part-solid nodules. The absolute difference value was significantly high in mediastinal windows, which means less similarity to pathologic size. The ICC values between CT and pathological measurement also tended to be higher with narrower $95 \%$ Cls in a lung window than those in mediastinal window. These results indicate the lung window is better than mediastinal window at predicting pathologic invasive component size $[6,18]$. A recent study by Yanagawa et al. suggested that the solid proportion in the lung window is more appropriate than using mediastinal window, because nodules with a larger solid proportion using the lung window tended to have greater malignant potential [19]. In addition, mediastinal windows tended to underestimate the size of pathologic invasive components compared with lung windows. This result is inevitable owing to various CT morphologies of invasive components, ranging from ground-glass to solid density. Invasive components manifesting as complete GGO to intermediate density on a CT scan may not appear on a mediastinal windows and lead to an underestimate $[6,18]$.

In addition to the inherent characteristics of invasive components being underestimated, we found that several characteristics of the lesions produce a greater difference in size measurements on mediastinal windows. A lesion with multiple scattered solid components and internal air densities was difficult to measure and showed increased interobserver variability on the mediastinal window compared with the lung window (Fig. 3). Also, multiplicity of solid components caused increased interobserver variability, especially in the mediastinal window (Fig. 4). Current IASLC guidelines recommend measuring the long axis of the largest solid component in the case of part-solid lesions with several solid components [1]. Although we measured and added all the solid components in order to measure as closely as possible the pathologic invasive components, we still encountered underestimates. Kim et al. reported substantial interobserver and intraobserver variability in determining the multiplicity and size of the solid components, and that the multiplicity of the solid component was not a significant risk factor of tumor recurrence [20]. Our study also demonstrated that the sum of multiple solid components is prone to observer variability and could be inaccurate.

Page $11 / 17$ 
Our study had several limitations. First, it was retrospective; therefore, CT protocols and section thickness were not uniform. Second, the pathological assessment we used as a reference standard may be inaccurate inadequately inflated lung tissue after resection with tissue processing.

\section{Conclusions}

\section{CT category for lung cancer staging}

In conclusion, lung window settings would be better for evaluating part-solid lung ADC, with comparable interobserver agreement and moderate accuracy for predicting pathologic invasive components. The considerable percentage (25\%) of discrepancies in categorizing solidity of the lesions may have decreased the accuracy of measurements. For accurate measurement and categorization of lung $A D C$, robust quantitative analysis is needed rather than a simple visual assessment.

\section{Abbreviations}

$\mathrm{ADC}$

lung adenocarcinoma

AIS

adenocarcinoma in situ

$\mathrm{Cl}$

confidence interval

GGN

ground-glass nodule

GGO

ground-glass opacity

ICC

intraclass correlation coefficient

MIA

minimally invasive adenocarcinoma

SD

standard deviation

\section{Declarations}

Ethical Approval and consent to participate

Institutional Review Board approval from Samsung Medical Center (SMC) was obtained. (IRB no. 201709-045)

Consent to publish 
Written informed consent was waived by the Institutional Review Board.

\section{Availability of data and materials}

The datasets used and/or analysed during the current study are available from the corresponding author on reasonable request.

\section{Competing interests}

The authors declare that they have no competing interest.

\section{Funding}

This research was supported by the Korea Health Technology R\&D Project through the Korea Health Industry Development Institute, which was funded by the Ministry of Health \& Welfare (HI17C0086) and by National Research Foundation of Korea grants funded by the Korean government (Ministry of Science, ICT, \& Future Planning) (No. NRF-2016R1A2B4013046 and NRF-2017M2A2A7A02018568). The funding body is not involved in the design of the study, collection and interpretation of data and writing the manuscript.

\section{Authors' contributions}

Conception and design: LHY, USW;

Acquisition of data: CY, PSG, KSH, KKH, CYS, PSG;

Analysis and interpretation of data: $\mathrm{CY}, \mathrm{KSH}, \mathrm{CYS}, \mathrm{SI}, \mathrm{KHS}$;

Writing, review and critical revision of the manuscript: All authors

\section{Acknowledgements}

Not applicable

\section{References}

1. Travis WD, Asamura H, Bankier AA, Beasley MB, Detterbeck F, Flieder DB, Goo JM, MacMahon H, Naidich D, Nicholson AG et al: The IASLC Lung Cancer Staging Project: Proposals for Coding T Categories for Subsolid Nodules and Assessment of Tumor Size in Part-Solid Tumors in the Forthcoming Eighth Edition of the TNM Classification of Lung Cancer. Journal of thoracic oncology : official publication of the International Association for the Study of Lung Cancer 2016, 11(8):12041223.

2. Hwang EJ, Park CM, Ryu Y, Lee SM, Kim YT, Kim YW, Goo JM: Pulmonary adenocarcinomas appearing as part-solid ground-glass nodules: is measuring solid component size a better prognostic 
indicator? European radiology 2015, 25(2):558-567.

3. Travis WD BE, Noguchi M, et al: The New IASLC/ATS/ERS international multidisciplinary lung adenocarcinoma classification. Journal of thoracic oncology : official publication of the International Association for the Study of Lung Cancer 2011, 6:244-285.

4. Lee KH, Goo JM, Park SJ, Wi JY, Chung DH, Go H, Park HS, Park CM, Lee SM: Correlation between the size of the solid component on thin-section CT and the invasive component on pathology in small lung adenocarcinomas manifesting as ground-glass nodules. Journal of thoracic oncology : official publication of the International Association for the Study of Lung Cancer 2014, 9(1):74-82.

5. Lee SM, Goo JM, Lee KH, Chung DH, Koh J, Park CM: CT findings of minimally invasive adenocarcinoma (MIA) of the lung and comparison of solid portion measurement methods at CT in 52 patients. European radiology 2015, 25(8):2318-2325.

6. Ahn H, Lee KW, Lee KH, Kim J, Kim K, Chung JH, Lee CT: Effect of computed tomography window settings and reconstruction plane on 8th edition T-stage classification in patients with lung adenocarcinoma manifesting as a subsolid nodule. European journal of radiology 2018, 98:130-135.

7. Ridge CA, Yildirim A, Boiselle PM, Franquet T, Schaefer-Prokop CM, Tack D, Gevenois PA, Bankier AA: Differentiating between Subsolid and Solid Pulmonary Nodules at CT: Inter- and Intraobserver Agreement between Experienced Thoracic Radiologists. Radiology 2016, 278(3):888-896.

8. Tsutani Y, Miyata Y, Mimae T, Kushitani K, Takeshima Y, Yoshimura M, Okada M: The prognostic role of pathologic invasive component size, excluding lepidic growth, in stage I lung adenocarcinoma. The Journal of thoracic and cardiovascular surgery 2013, 146(3):580-585.

9. Maeyashiki T, Suzuki K, Hattori A, Matsunaga T, Takamochi K, Oh S: The size of consolidation on thin-section computed tomography is a better predictor of survival than the maximum tumour dimension in resectable lung cancer. European journal of cardio-thoracic surgery : official journal of the European Association for Cardio-thoracic Surgery 2013, 43(5):915-918.

10. Tsutani Y, Miyata Y, Nakayama H, Okumura S, Adachi S, Yoshimura M, Okada M: Prognostic significance of using solid versus whole tumor size on high-resolution computed tomography for predicting pathologic malignant grade of tumors in clinical stage IA lung adenocarcinoma: a multicenter study. The Journal of thoracic and cardiovascular surgery 2012, 143(3):607-612.

11. van Riel SJ, Sanchez CI, Bankier AA, Naidich DP, Verschakelen J, Scholten ET, de Jong PA, Jacobs C, van Rikxoort E, Peters-Bax L et al: Observer Variability for Classification of Pulmonary Nodules on Low-Dose CT Images and Its Effect on Nodule Management. Radiology 2015, 277(3):863-871.

12. Hansell DM, Bankier AA, MacMahon H, McLoud TC, Muller NL, Remy J: Fleischner Society: glossary of terms for thoracic imaging. Radiology 2008, 246(3):697-722.

13. Naidich DP, Bankier AA, MacMahon H, Schaefer-Prokop CM, Pistolesi M, Goo JM, Macchiarini P, Crapo JD, Herold CJ, Austin JH et al: Recommendations for the management of subsolid pulmonary nodules detected at CT: a statement from the Fleischner Society. Radiology 2013, 266(1):304-317.

14. Son JY, Lee HY, Kim JH, Han J, Jeong JY, Lee KS, Kwon OJ, Shim YM: Quantitative CT analysis of pulmonary ground-glass opacity nodules for distinguishing invasive adenocarcinoma from non- 
invasive or minimally invasive adenocarcinoma: the added value of using iodine mapping. European radiology 2016, 26(1):43-54.

15. Ikeda K, Awai K, Mori T, Kawanaka K, Yamashita Y, Nomori H: Differential diagnosis of ground-glass opacity nodules: CT number analysis by three-dimensional computerized quantification. Chest 2007, 132(3):984-990.

16. Gong J, Liu J, Hao W, Nie S, Wang S, Peng W: Computer-aided diagnosis of ground-glass opacity pulmonary nodules using radiomic features analysis. Physics in medicine and biology 2019, 64(13):135015.

17. Ganeshan B, Abaleke S, Young RC, Chatwin CR, Miles KA: Texture analysis of non-small cell lung cancer on unenhanced computed tomography: initial evidence for a relationship with tumour glucose metabolism and stage. Cancer imaging : the official publication of the International Cancer Imaging Society 2010, 10:137-143.

18. Yoo RE, Goo JM, Hwang EJ, Yoon SH, Lee CH, Park CM, Ahn S: Retrospective assessment of interobserver agreement and accuracy in classifications and measurements in subsolid nodules with solid components less than 8mm: which window setting is better? European radiology 2017, 27(4):1369-1376.

19. Yanagawa M, Kusumoto M, Johkoh T, Noguchi M, Minami Y, Sakai F, Asamura H, Tomiyama N: Radiologic-Pathologic Correlation of Solid Portions on Thin-section CT Images in Lung Adenocarcinoma: A Multicenter Study. Clinical lung cancer 2018, 19(3):e303-e312.

20. Kim H, Goo JM, Suh YJ, Hwang EJ, Park CM, Kim YT: Measurement of Multiple Solid Portions in PartSolid Nodules for T Categorization: Evaluation of Prognostic Implication. Journal of thoracic oncology : official publication of the International Association for the Study of Lung Cancer 2018, 13(12):1864-1872.

\section{Figures}
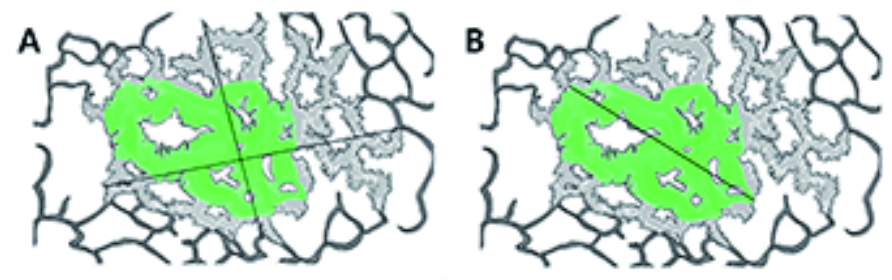

Figure 1

Schematic drawings for measurement of lung adenocarcinoma manifesting as a part-solid nodule on CT (a) Measurement of maximum and perpendicular diameters of the total lesion (black line) compared with total area on pathology (gray area). (b) Measurement of maximal diameter of the solid component (black line) compared with the pathologic invasive component (green area). 


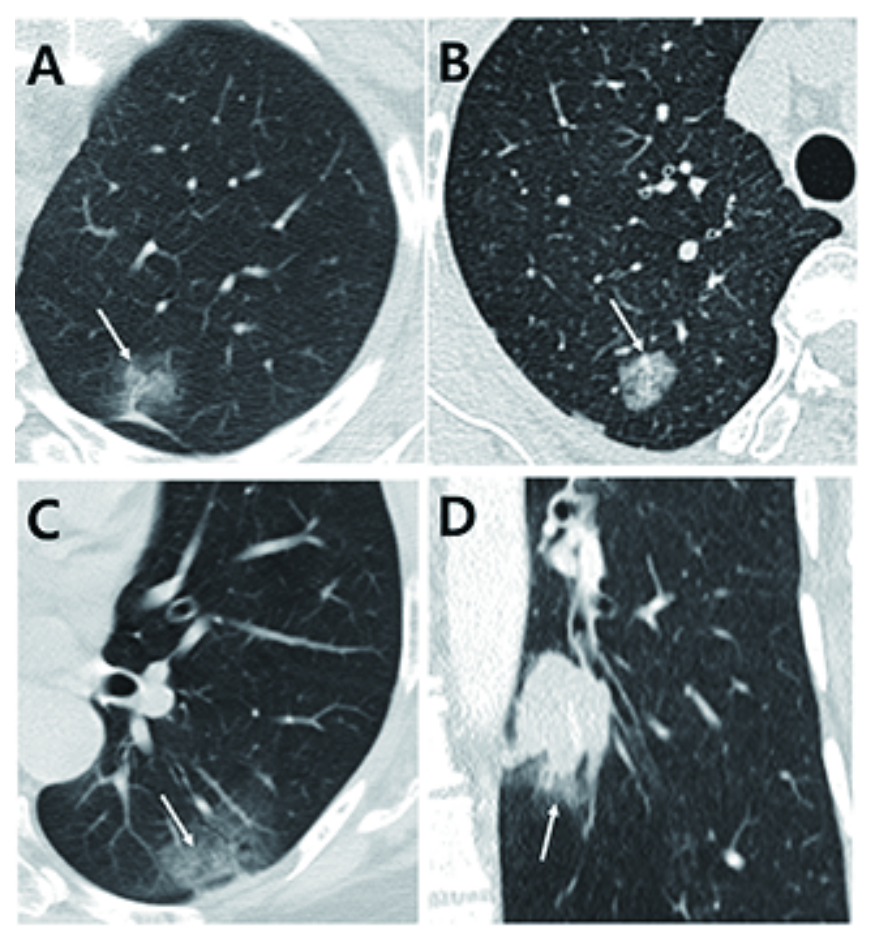

Figure 2

Atypical cases causing discrepancy between observers in categorizing solidity In cases where pure-GGN and part-solid nodule need to be distinguished, there were three patterns of atypical cases (a-c): (a) lesion with borderline attenuation not well fit to pure-GGN nor part-solid lesion, (b) entirely heterogeneous attenuated lesion, and (c) lesion with gradually smooth transitional margin. In cases where part-solid and solid nodules need to be distinguished, the predominant solid mass with small area of adjacent GGO (d) was the main cause of discrepancy.

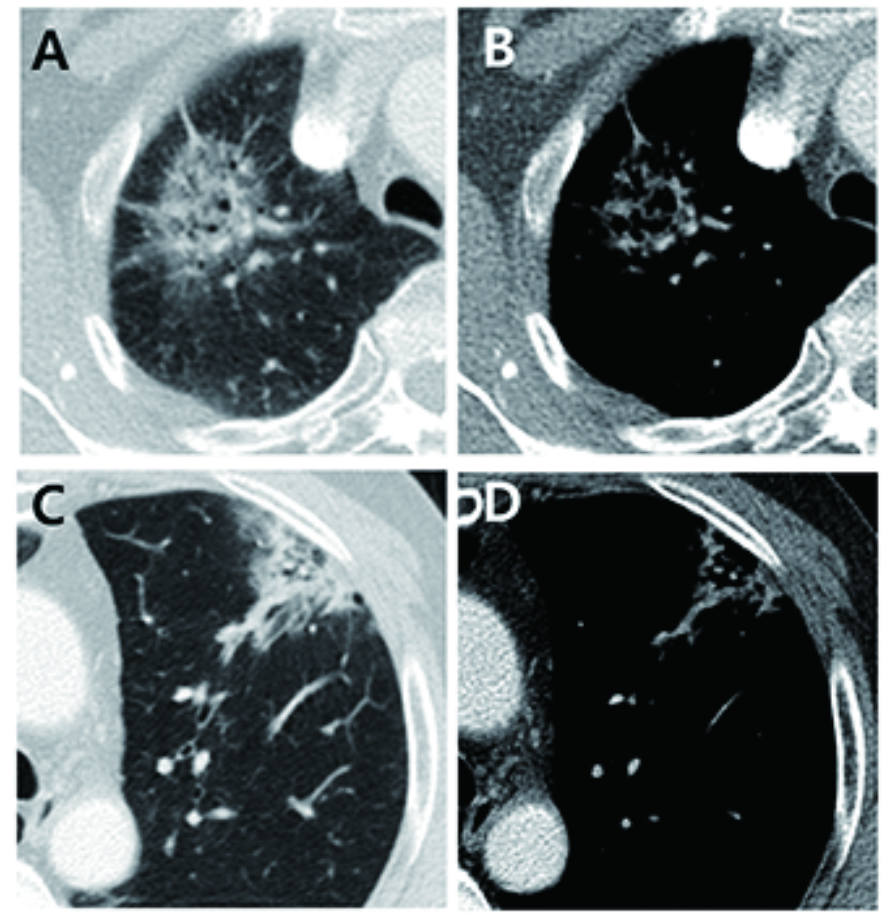

Figure 3 
Case with lower interobserver agreement for CT measurement on mediastinal windows. (a-b) A moderately differentiated ADC, acinar pattern, with a $28 \mathrm{~mm}$ invasive component (T1c). Part-solid nodule with a spiculated margin is noted in the right upper lobe on the lung window setting (a); on mediastinal windows, multiple internal air densities and air bronchogram make it difficult to measure solid components of the nodule (b). (c-d) with moderately differentiated ADC, acinar and lepidic pattern, with a $25 \mathrm{~mm}$ invasive component (T1c). On lung windows, an irregular subpleural part-solid nodule with an adjacent bronchovascular bundle is demonstrated (c). On mediastinal windows, both irregular margin and internal air densities make it difficult to measure the solid component of the lesion (d).
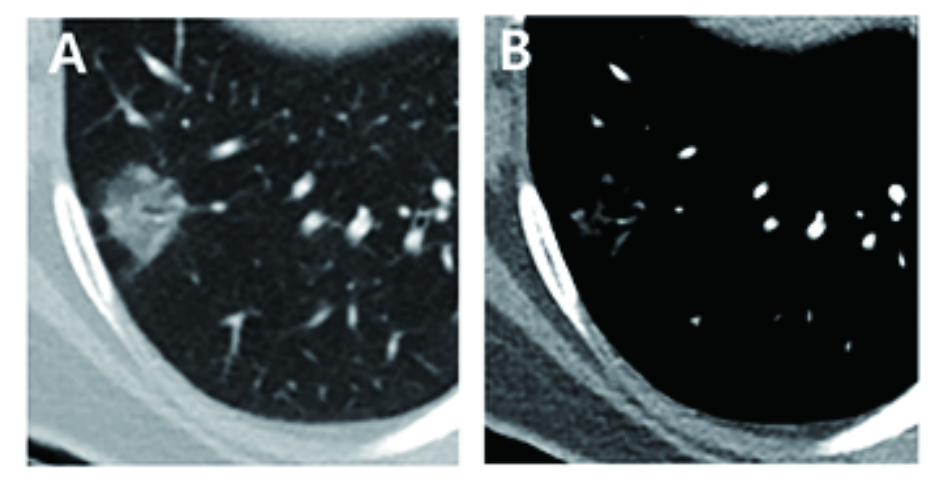

Figure 4

A moderately differentiated ADC, acinar and papillary pattern, with a $17 \mathrm{~mm}$ invasive component (T1b). (a-b) Demonstrates part-solid nodule with several solid components. (a) On lung windows, part-solid nodules with several dense solid components is demonstrated. An internal bronchovascular structure is noted. (b) On mediastinal windows, several small nodular or linear solid components and internal branching vascular structure make it difficult to measure the maximal diameter of a solid component. 